\title{
A feasibility study of laparoscopic sentinel lymph node mapping by cervical tracer injection in endometrial cancer
}

Chisa Shimada, Yukiharu Todo, Hiroyuki Yamazaki, Shinichiro Minobe, Hidenori Kato Division of Gynecologic Oncology, National Hospital Organization, Hokkaido Cancer Center, 4-2 Kikusui, Shiroishi-ku, Sapporo 003-0804, Japan

\section{Objective}

This study aimed to verify the feasibility of laparoscopic approach for sentinel lymph node (SLN) surgery in endometrial cancer.

\section{Design}

We retrospectively enrolled 118 patients with endometrial cancer who had undergone SLN mapping, using technetium colloid ( $\mathrm{Tc}^{99 \mathrm{~m}}$ ) and/or indocyanine green (ICG) injected into the uterine cervix with an intraoperative gamma-detecting probe and/or photodynamic eye camera system to locate hot spots. SLN detection rate (unilateral or bilateral) and incidence of complications were compared between open approach (O group, $n=65$ ) and laparoscopic approach (L group, $n=53)$.

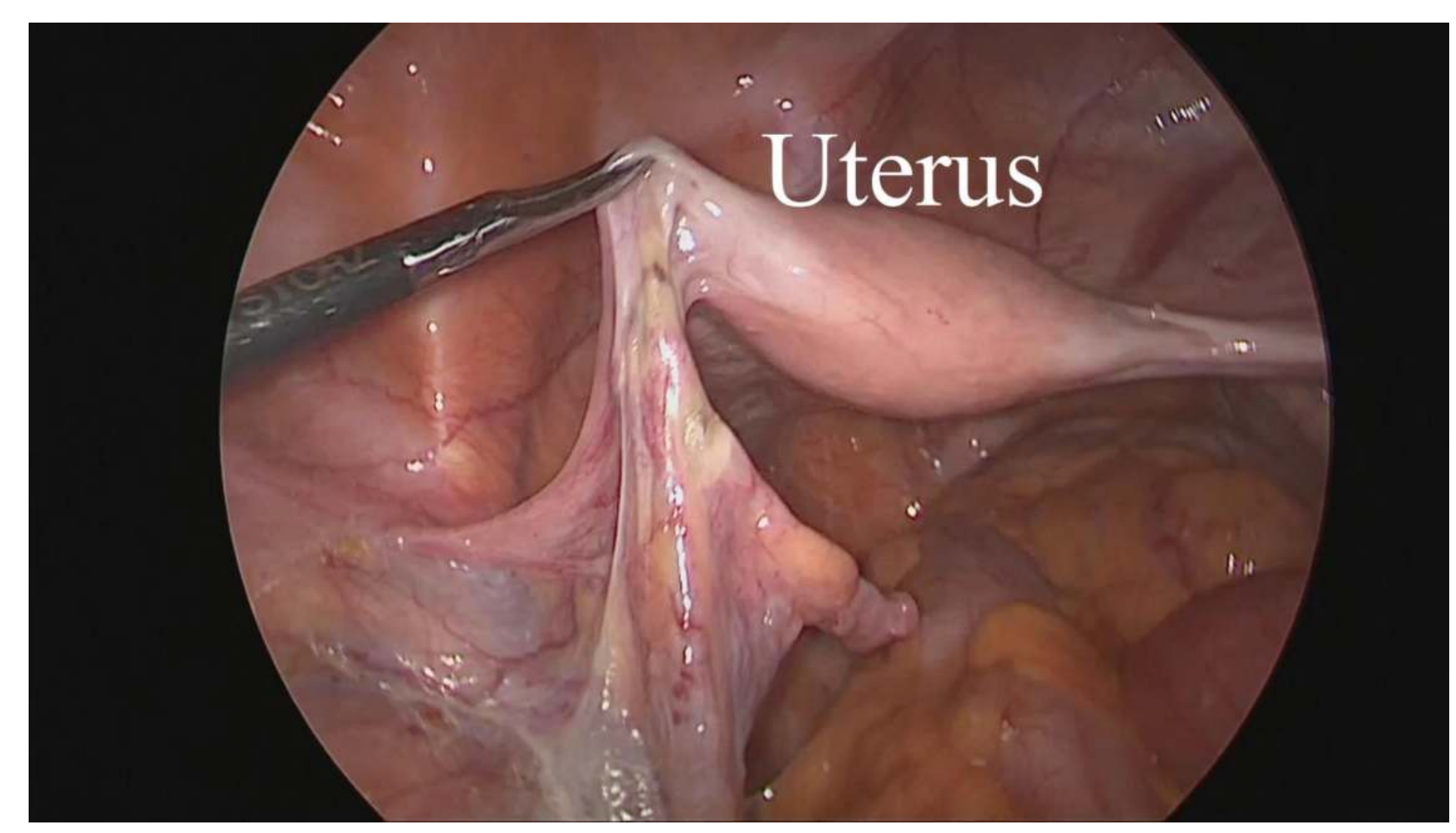

\section{Results}

Patient backgraoud

\begin{tabular}{lc}
\hline Age: median & $60(36-84)$ \\
BMI : median & $23.4(16.8-47.6)$ \\
FIGO stage & $101(85.6 \%)$ \\
IA & $8(6.8 \%)$ \\
IB & $1(0.8 \%)$ \\
II & $2(1.7 \%)$ \\
IIIA & $4(3.4 \%)$ \\
IIIC1 & $2(1.7 \%)$ \\
IIIC2 & \\
Histrogical type & $84(71.2 \%)$ \\
endometrioid G1 & $20(16.9 \%)$ \\
endometrioid G2 & $3(2.5 \%)$ \\
endometrioid G3 & $8(6.8 \%)$ \\
clear/serous & $3(2.5 \%)$ \\
\hline
\end{tabular}

Performance of surgery

\begin{tabular}{lc}
\hline approach & \\
$\quad$ laparotomy & $65(55.1 \%)$ \\
$\quad$ laparoscopic & $53(44.9 \%)$ \\
systematic lyphadectomy & $69(58.5 \%)$ \\
para aortic lymph node isolated & $24(20.3 \%)$ \\
operation time:median & $173(95-510) \mathrm{min}$ \\
blood loss:median & $75(2-918) \mathrm{cc}$ \\
number of isolated lymph node:median & $12(1--110)$ \\
pelvic & $12(1--82)$ \\
para-aortic & $0(0-28)$ \\
\hline
\end{tabular}

Tracers and SLN detection ability

\begin{tabular}{cc}
\hline tracer & \\
two kinds & $39(33.1 \%)$ \\
single & $79(66.9 \%)$ \\
Tc99m & $\mathrm{X}$ \\
ICG & $\mathrm{X}$ \\
SLN detection & \\
bilateral & $91(77.1 \%)$ \\
unirateral & $19(16.1 \%)$ \\
no detection & $8(6.8 \%)$ \\
\hline
\end{tabular}

Perioperative complication

\begin{tabular}{lc}
\hline no & $103(87.3 \%)$ \\
yes & $15(12.7 \%)$ \\
dysria & $4(3.4 \%)$ \\
lower limb lymphedema & $3(2.5 \%)$ \\
deep-vein thrombosis & $2(1.7 \%)$ \\
ileus & $1(0.8 \%)$ \\
obturator nerve paralysis & $1(0.8 \%)$ \\
pelvic peritonitis & $1(0.8 \%)$ \\
renal dysfunctionn & $1(0.8 \%)$ \\
wound dehiscence & $1(0.8 \%)$ \\
\hline
\end{tabular}

\begin{tabular}{|c|c|c|c|}
\hline $\begin{array}{c}\text { combinasion } \\
\text { with two kids of tracer }\end{array}$ & $49 \%$ & $20 \%$ & $\mathrm{p}<0.001$ \\
\hline successful mapping rate & $8 \%$ & $6 \%$ & $\mathrm{p}=0.76$ \\
\hline complication rate & $6 \%$ & $18 \%$ & $\mathrm{p}<0.05$ \\
\hline
\end{tabular}

\section{Conclusion}

Laparoscopic approach might obtain a SLN detection ability equal to open approach without increasing the number of perioperative complication despite limitation of the present study, i.e. a combination with two kinds of tracers might have given the $L$ group an advantage over the $\mathrm{O}$ group. 